\title{
Characterization of radish mitochondrial atp $A$ : influence of nuclear background on transcription of atp $A$-associated sequences and relationship with male sterility
}

\author{
Christopher A. Makaroff, ${ }^{1}$ Ingrid J. Apel ${ }^{2}$ and Jeffrey D. Palmer ${ }^{3}$ \\ Department of Biology. The University of Michigan, Ann Arbor, MI 48109, USA; (Present addresses: \\ ${ }^{1}$ Department of Chemistry, Miami University, Oxford, OH 45056, USA; ${ }^{2}$ Department of Internal \\ Medicine, University of Michigan School of Medicine, Ann Arbor, MI 48109, USA; ${ }^{3}$ Department of \\ Biology, Indiana University, Bloomington, IN 47405, USA)
}

Received 12 June 1990; accepted in revised form 3 August 1990

Key words: cytoplasmic male sterility, mitochondrial DNA, nuclear-mitochondrial interactions, Ogura cytoplasm, rearrangements

\begin{abstract}
We have previously shown that the mitochondrial gene atpA, encoding the $\alpha$ subunit of $\mathrm{F}_{1}$ ATP synthase, is associated with DNA rearrangements and nuclear-specific transcript patterns in the male-sterile cytoplasm of Ogura radish. Here we present a detailed characterization of this gene from both the normal (fertile) and Ogura (male-sterile) cytoplasms of radish to determine if it is involved in Ogura cytoplasmic male sterility. The normal and Ogura radish atp $A$ loci are virtually identical for $3.8 \mathrm{~kb}$, including a 507 codon open reading frame whose, product is approximately $92 \%$ identical to other plant ATPA polypeptides. Rearrangement breakpoints have been identified $613 \mathrm{bp} 5^{\prime}$ and $1663 \mathrm{bp} 3^{\prime}$ to the atp $A$ coding region. The $5^{\prime}$ rearrangement breakpoint is located within a repeated sequence that has been associated with other rearrangement events in radish mitochondria. The previously identified transcript difference results from transcription originating upstream of this rearrangement site. Although the presence of this transcript is affected by nuclear background, analyses in several different sterile and fertile nuclear backgrounds indicate that the presence of this transcript is not strictly correlated with male sterility. In addition, normal levels of ATPA polypeptide are present in sterile plants containing the Ogura cytoplasm.
\end{abstract}

\section{Introduction}

Cytoplasmic male sterility (CMS) is a maternally inherited trait that is characterized by a plant's inability to produce functional pollen. Malesterile cytoplasms have long been of interest because of their use in hybrid seed production; however, they also provide a useful system in which to study mitochondrial function and nuclear-mitochondrial interactions. There are numerous examples of alterations in mitochondrial DNA (mtDNA) structure and mitochondrial RNA (mtRNA) and protein patterns associated with CMS plants [reviewed in 21, 22]. Nuclear loci that suppress these mitochondrial alterations and restore fertility to CMS cytoplasms have also been identified.

The Ogura cytoplasm of radish (Raphanus 
sativus) was the first example of CMS in a crucifer (family Brassicaceae). Radish plants containing the Ogura cytoplasm develop normally until early microspore formation when a collapse of the tapetal tissue leads to microspore degeneration [29]. Three nuclear loci have been identified in European cultivars of radish that may be involved in fertility restoration of the Ogura cytoplasm in Brassica napus [14]. However, Pelletier et al. [32] have suggested that the male sterility observed for the $B$. napus-Ogura cytoplasm combination may be the result of both mitochondrial alterations (observed in a radish nuclear background) and nuclear-mitochondrial incompatibilities (alloplasmic male sterility). Therefore, it is not clear how many nuclear loci are necessary to restore fertility to the Ogura cytoplasm in radish.

The mitochondrial genome of Ogura radish is highly rearranged relative to, and contains sequences not present in, the normal radish genome [25]. In addition, altered transcript patterns have been identified for three genes: atpA, atp6, and coxI. Of these genes, only the atp $A$ transcript pattern is affected by nuclear background. In order to identify the mitochondrial alteration(s) associated with Ogura CMS, we have characterized the Ogura mitochondrial genome further, including the detailed analyses of these three genes. Altered atp6 transcript patterns are the result of a complex series of rearrangements that have resulted in an Ogura-specific transcriptional unit containing a 105 codon open reading frame as the first gene of a bicistronic mRNA. In addition, multiple nucleotide differences in the Ogura atp6 5' flanking region and coding sequence are predicted to eliminate normal translation of atp 6 [24]. In contrast, characterization of the normal and Ogura radish coxI loci indicates that coxI is not involved in Ogura CMS and that the observed differences in coxI transcript patterns are the result of a rearrangement that has occurred in normal radish (Makaroff, Apel and Palmer, unpublished).

We report here a detailed analysis of the atpA locus from both normal and Ogura radish. The previously identified transcript difference is the result of transcription that originates upstream of a 5' rearrangement breakpoint at Ogura atpA. Analysis of Ogura atp $A$ transcript patterns in several different nuclear backgrounds indicates that while the presence of this transcript is dependent on nuclear background, it is not strictly correlated with male sterility. Furthermore, normal levels of ATPA are present in sterile and fertile plants containing the Ogura cytoplasm, indicating that atpA is not causally related to CMS.

\section{Materials and methods}

\section{Plant material}

Raphanus sativus cv. Scarlet Knight (wild-type radish) was the source of the normal radish cytoplasm. The mitochondrial genome of Scarlet Knight exhibits the same restriction enzyme profile as those of three other commonly used male-fertile radish cultivars ([30], Makaroff and Palmer, unpublished) and thus is used to represent what we term the 'normal' cytoplasm of radish. The rapid-cycling radish line $\mathrm{CrGC15}$ (R1rr; obtained from the Crucifer Genetics Cooperative) was the primary source of the Ogura cytoplasm. Three other sources of the Ogura cytoplasm used in this study were: 1) an open-pollinated white radish line (Iwate) that segregates approximately $50: 50$ for fertile and sterile plants; 2) a $B$. napus line containing the Ogura cytoplasm (male-sterile) (this and the preceding line were obtained from Dr. J. Imamura, Plantech Research Institute, Japan); and 3) a second openpollinated white radish line (5241) that segregates for fertile and sterile plants (50:50) (obtained from Dr. S. Magnuson, Harris Moran Seed Co., CA). Sterile and fertile (nuclear-restored) plants containing the Ogura cytoplasm were distinguished by their ability to produce pollen.

\section{Isolation and labeling of nucleic acids}

Mitochondrial DNA was isolated from green leaves of both normal radish and plants contain- 
ing the Ogura cytoplasm (CrGC15, Iwate and B. napus) by the DNase I procedure [18]. Mitochondrial RNA was isolated in the presence of aurintricarboxylic acid from mitochondria purified by differential centrifugation from green leaves (normal radish) and green leaves and flowers (Ogura cytoplasm) [38]. Plasmid DNA was isolated using the alkaline lysis procedure [3] and purified over $\mathrm{CsCl}$ gradients as necessary. Probes used in S1 nuclease mapping and northern and Southern hybridization experiments were labeled using common techniques [27], including primer extension of single-stranded DNA, nick translation and 5 ' - or 3 '-end labeling with T4 kinase or Klenow fragment, respectively, followed by purification by gel electrophoresis. Single-stranded molecules for the 277 bp Eco RI and 228 bp $B g l \mathrm{II}-\mathrm{Spe}$ I fragments were isolated on strand separation gels [27] and their identity (coding vs noncoding strand) determined by northern analysis.

\section{DNA sequence analysis}

Normal radish atpA was previously localized to sequences spanning SalI fragments of 3.4 and $13.6 \mathrm{~kb}$ [25]. Most of the normal atpA locus was subcloned as a $2.8 \mathrm{~kb}$ Sal I-Hind III fragment from the previously isolated $3.4 \mathrm{~kb} \mathrm{Sal} \mathrm{I} \mathrm{fragment}$ [31]. The $5^{\prime}$ coding and flanking regions were isolated as a $1.2 \mathrm{~kb} \mathrm{Bam} \mathrm{HI} \mathrm{fragment} \mathrm{by} \mathrm{colony}$ hybridization screening [27] of a normal radish mtDNA library with a $0.4 \mathrm{~kb}$ Bam HI-Sal I fragment internal to Ogura atpA (Fig. 1). The Ogura radish atpA locus was subcloned as $1.25 \mathrm{~kb} N r u \mathrm{I}-$ $S a l$ I and $3.45 \mathrm{~kb}$ Sal I-Bam HI fragments from previously isolated $12.0 \mathrm{~kb}$ and $9.6 \mathrm{~kb} \mathrm{Sal} \mathrm{I} \mathrm{frag-}$ ments, respectively [25].

Standard techniques were used in the construction and analysis of recombinant clones [27]. Dideoxy sequencing [34] of exonuclease III[11] and restriction enzyme-deleted clones with vector- and atpA-specific oligonucleotides was conducted as described [24]. DNA sequences were analyzed on a Sun Microsystems, Inc. minicomputer using the Eugene program (Department of Cell Biology, Baylor College of Medicine, Houston, TX).

\section{Sl nuclease mapping}

Transcript mapping experiments were conducted using the procedure of Berk and Sharp [2]. RNA $(10 \mu \mathrm{g})$ was co-precipitated together with a DNA probe (Fig. 4), resuspended in $20 \mu$ of hybridization buffer ( $40 \mathrm{mM}$ PIPES, pH 6.4, $1 \mathrm{mM}$ EDTA, $0.4 \mathrm{M} \mathrm{NaCl}$, and $80 \%$ formamide), denatured at $80^{\circ} \mathrm{C}$ for $5 \mathrm{~min}$, and allowed to anneal for $12-16 \mathrm{~h}$ at $45-56{ }^{\circ} \mathrm{C}$ (the GC content of the fragment determined the annealing temperature). Samples were treated with $300 \mu \mathrm{l}$ of a buffer containing $250 \mathrm{mM} \mathrm{NaCl}, 1 \mathrm{mM} \mathrm{ZnSO}, 30 \mathrm{mM}$ potassium acetate, $\mathrm{pH} 4.5$ and $5 \%(\mathrm{v} / \mathrm{v})$ glycerol for $60 \mathrm{~min}$ at $37^{\circ} \mathrm{C}$. The samples were then phenol-extracted, ethanol-precipitated, resuspended in denaturing stop dye and electrophoresed on both $5 \%$ polyacrylamide $/ 8 \mathrm{M}$ urea gels and high-resolution $6 \%$ polyacrylamide $/ 8 \mathrm{M}$ urea gels next to ${ }^{32} \mathrm{P}$-labeled Bluescript $\mathrm{KS}$ digested with

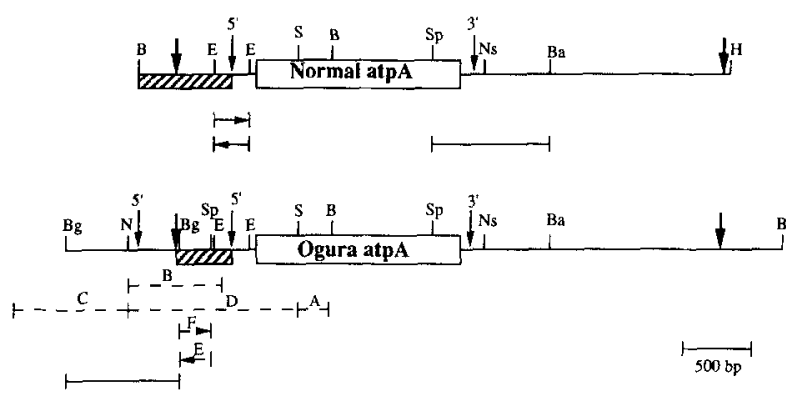

Fig. 1 . Organization of the atpA loci from the normal and Ogura cytoplasms of radish. The atpA locus of normal radish (top) is shown aligned with that of Ogura radish (bottom). Open boxes represent the atp $A$ coding regions; shaded boxes represent sequences present elsewhere in the radish genomes. Rearrangement breakpoints are marked with heavy vertical arrows. Fragments used as probes in nuclease mapping experiments and northern hybridizations are shown as solid and dashed lines, respectively. Single-stranded probes are shown as horizontal arrows. Transcript termini are denoted with light vertical arrows. Selected restriction sites, which were used in the construction of probes and fusion proteins, are shown as: B, Bam $\mathrm{HI} ; \mathrm{Ba}, \mathrm{Bal}$; $\mathrm{Bg}$, BglII; E, Eco RI; H, Hind III; N, Nru I; Ns, Nsi I; S, SalI; Sp, Spe I. 


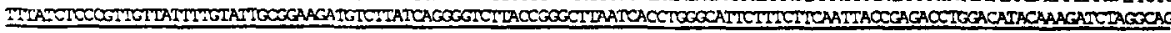

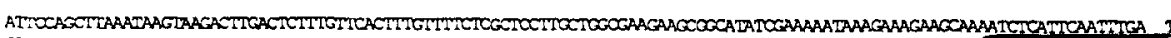

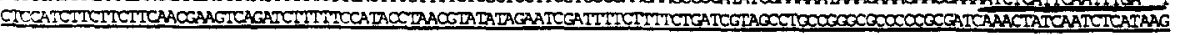

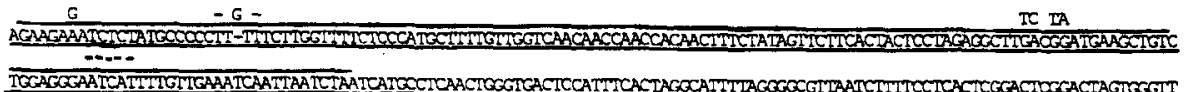

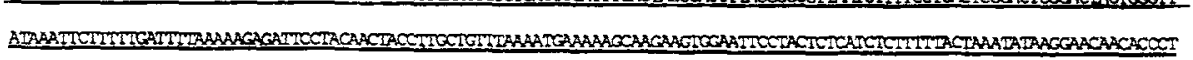

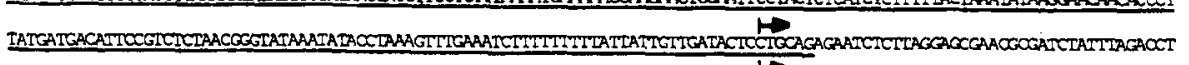

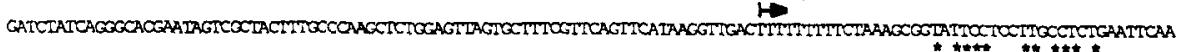

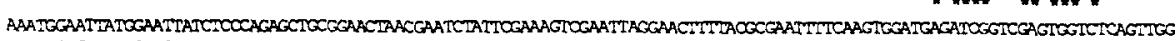

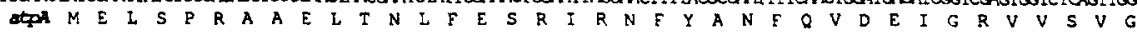

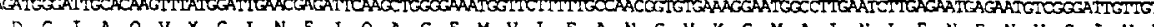
$\begin{array}{lllllllllllllllllllllllllllllllllllllll}D & G & I & A & Q & V & Y & G & L & N & E & I & Q & A & G & E & M & V & L & F & A & N & G & V & K & G & M & A & L & N & L & E & N & E & N & V & G & I & V\end{array}$

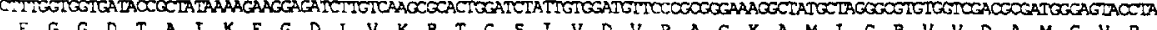
$\begin{array}{llllllllllllllllllllllllllllllllllllllll}F & G & G & D & I & A & I & K & E & G & D & L & V & K & R & I & G & S & I & V & D & V & P & A & G & K & A & M & I & G & R & V & V & D & A & M & G & V & P & 1\end{array}$

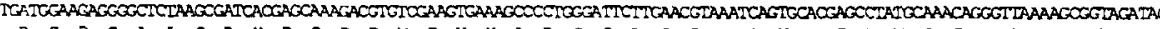
$\begin{array}{llllllllllllllllllllllllllllllllllllllll}D & G & R & G & A & L & S & D & H & E & Q & R & R & V & E & V & K & A & P & G & I & L & E & R & K & S & V & H & E & P & M & Q & I & G & L & K & A & V & D & S\end{array}$

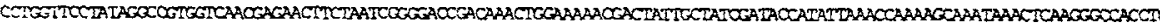

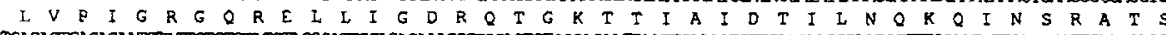

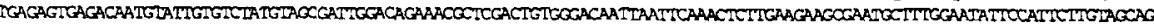

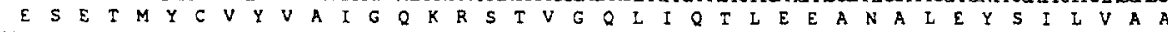

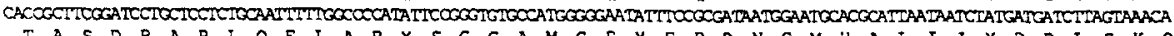

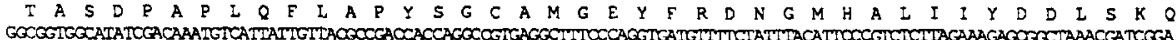

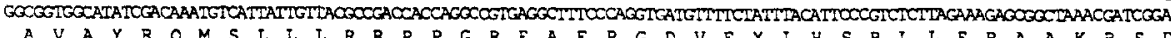

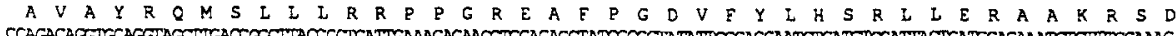

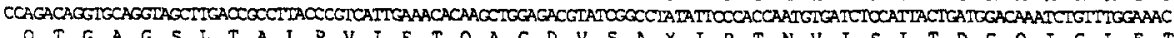

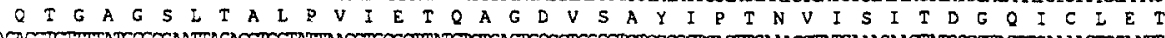

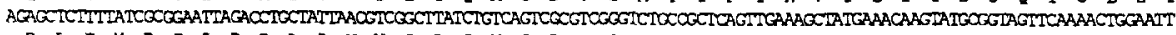

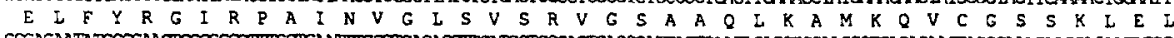

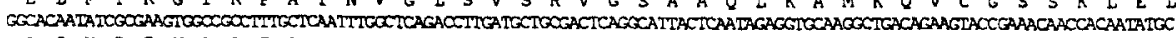
$A Q$ Y R E V A A E A Q F G S D L D A A T Q A L L N R G A R I I E V P K Q P Q Y A ACTACTTC

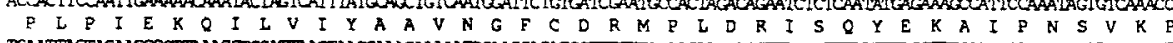

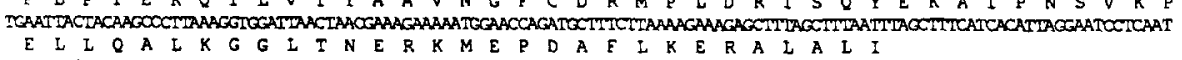

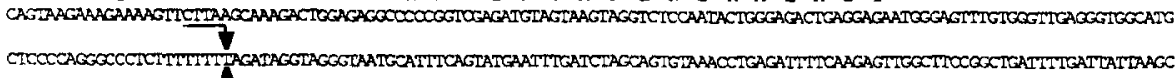

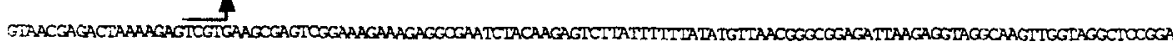

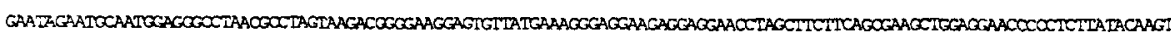

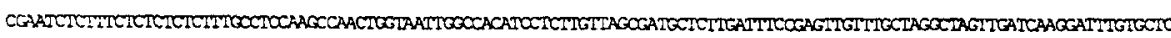

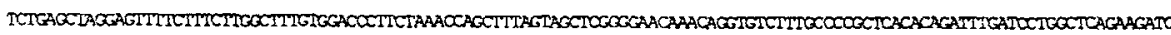
COAATACCGACGACAAACAATAACACAAACCAMTCOACCTOCAAAAGATCAOCAATAAAAGGAAGATIACAGCGAAGGAAGAGACGAATAGIATGATGIACGGTAAGGGATAGCIGIT

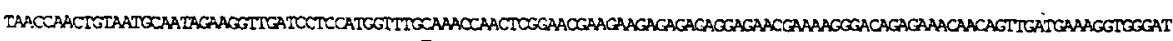

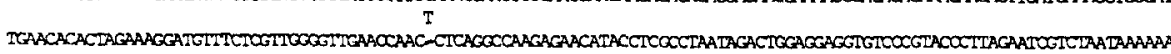

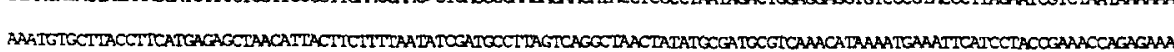

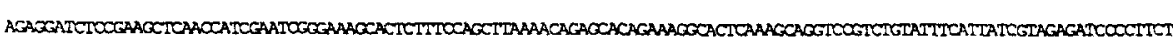

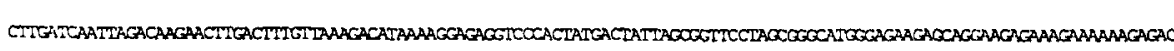

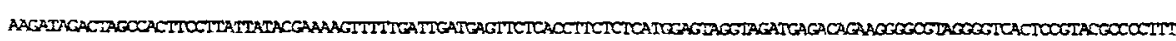

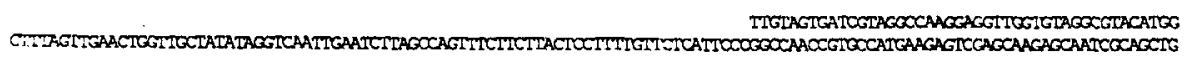

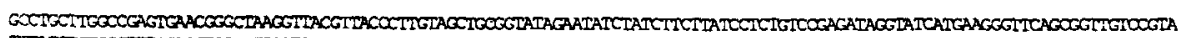
TITATCTCTCOCTITCAOCTCOCATTOCTCOCTTOCTISTAAAAGCIT

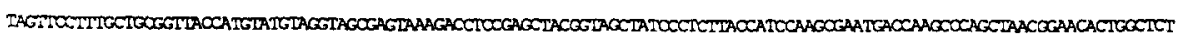

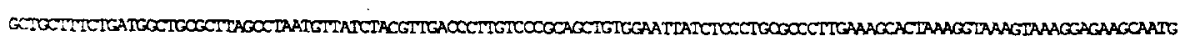

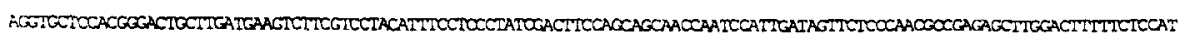

Fig. 2. Nucleotide and derived amino acid sequences of the atp $A$ loci from normal and Ogura radish. The Ogura sequence (top line) is aligned with the normal sequence (bottom line). Where the two sequences are identical only the normal sequence is shown. Insertions/deletions relative to normal atp $A$ within the aligned regions are represented by a dash ( - ). Numbering of nucleotides is from the predicted translation start of atp A. A $689 \mathrm{bp}$ sequence present $5^{\prime}$ to normal radish coxI-2 is underlined; a $175 \mathrm{bp}$ sequence present $5^{\prime}$ to the Ogura orf105-atp 6 locus is overlined. Nucleotides complimentary to the $18 \mathrm{~S}$ rRNA, allowing for G-U 
HinfI and dideoxy sequencing ladders, respectively.

\section{Northern and Southern blot analysis}

Conditions for the electrophoresis, transfer and hybridization of RNA ( $7.5 \mu \mathrm{g} /$ lane) to ${ }^{32} \mathrm{P}$-labeled probes were as described previously [26]. Ridosomal RNAs and Hae III fragments of phage $\Phi \times 174$ were used as size standards. Probe used in hybridization experiments are described in Fig. 3. Standard techniques were used in the construction of Southern blots and their hybridization to nick-translated probes [27].

\section{Antibody production and western analysis}

A 993 bp Bam HI-Nsi I fragment of normal radish atpA (nucleotides 720 to 1713 ; Figs 1 and 3 ) was fused in-frame to Escherichia coli lac $Z$ in the vector pUR290 [31] to produce a $\beta$-galactosidase fusion protein containing the carboxy-terminal 262 amino acids of radish atpA. The fusion protein was overproduced in the presence of $1.0 \mathrm{mM}$ IPTG, purified twice on $10 \%$ SDS-polyacrylamide gels [19] and used to raise antibodies in New Zealand White rabbits following standard procedures [12]. A primary injection of protein $(200 \mu \mathrm{g})$ in Freund's complete adjuvant was given, followed by two boosters $(200 \mu \mathrm{g}$ protein each in Freund's incomplete adjuvant) at sixweek intervals. Serum was collected to weeks after the second boost. Prior to use, serum containing ATPA antibodies was treated with acetone-extracted proteins prepared from $E$. coli cells containing the plasmid pUR290. Preimmune serum when used in western hybridizations did not cross-react with the $55 \mathrm{kDa}$ mitochondrial protein (data not shown). Antibody to maize coxII and the spinach chloroplast $32 \mathrm{kDa}$ protein was kindly provided by C. S. Levings III and N. Bowlby, respectively.
Mitochondria were isolated from radish leaves by differential centrifugation and purified over Percoll gradients [20]. Mitochondrial proteins were separated on $12.5 \%$ SDS-polyacrylamide gels [19] and transferred to nitrocellulose using the procedure of Towbin [39]. Western blots were treated with primary antibodies overnight and developed using a Bio-Rad Immuno-Blot GoatAnti-Rabbit Alkaline Phosphatase assay kit under conditions recommended by the supplier. Protein content was determined as previously described [23].

\section{Results}

Rearrangements and novel sequences are associated with atp $A$ in Ogura radish

The nucleotide sequence of a $4.1 \mathrm{~kb}$ segment of normal radish mtDNA that contains the atpA locus is shown in Fig. 2. Present in this sequence is a 507 codon open reading frame capable of encoding a $55113 \mathrm{Da}$ polypeptide, identified as atpA on the basis of DNA sequence identity. The derived amino acid sequence is $91-94 \%$ identical to that of maize [6], pea [28], Oenothera [35] and tobacco [7]. If however the atp $A$ transcript is edited, as has been observed for several other plant mitochondrial genes $[8,11,37]$, then the amino acid similarity may be different.

The nucleotide sequence of a $4.7 \mathrm{~kb}$ segment of Ogura radish mtDNA containing the atp $A$ locus is shown aligned with that of normal radish in Fig. 2. The two loci are virtually identical $(99.8 \%)$ for $3.8 \mathrm{~kb}$. Six nucleotide substitutions and five insertions/deletions are present in the common region. Rearrangement breakpoints between the two loci are located $613 \mathrm{bp} \mathrm{5^{ \prime }}$ and $1663 \mathrm{bp} 3^{\prime}$ to the atp $A$ coding region. The $5^{\prime}$ rearrangement breakpoint is located within a $689 \mathrm{bp}$ sequence (nucleotides -855 to -166 ) that is repeated elsewhere in the genome. Filter hybridizations indicate that part or all of this sequence is present at

basepairing, are indicated with an asterisk $\left(^{*}\right)$ under the corresponding sequence. Major $5^{\prime}$ transcript termini are indicated with horizontal arrows and $3^{\prime}$ transcript termini are indicated with vertical arrowheads (above the sequence for Ogura radish and below the sequence for normal radish). 
least four and six times in the normal and Ogura radish genomes, respectively (Makaroff, Apel and Palmer, unpublished). This dispersed repeat appears to be involved in homologous recombination events that generated a submolar form of the coxI gene. The boundaries of this repeat have been assigned based on a comparison with the coxI-associated repeat element. The sequences of additional members of this repeat family have not been determined, therefore the sizes of these repeat elements relative to the atp $A$ repeat may be different from that defined by the atp $A / c o x I$ comparison.

From the $5^{\prime}$ end of the sequenced region to nucleotide -613 the two atp $A$ loci are completely different. Southern hybridization experiments using Ogura mtDNA fragments from this region as probes indicate that this sequence is essentially absent from normal radish (data not shown). Multiple weak hybridization signals are, however, detected in both normal and Ogura mtDNAs, suggesting that this region contains one or more short, repeated sequences. Computer analysis of the Ogura mtDNA sequence from -1955 to +1 did not identify any open reading frames or similarity with known genes. Southern hybridizations indicate that this sequence is not derived from chloroplast DNA (data not shown), and therefore, the origin of this DNA sequence is unknown. These results are in agreement with earlier findings that the Ogura $12.0 \mathrm{~kb}$ Sal I fragment is, for the most part, not present in normal radish [6].

\section{Nuclear background affects the processing of atpA- related transcripts in Ogura radish}

A series of transcript mapping experiments was conducted in order to characterize a previously identified $2700 \mathrm{nt}$ atpA transcript, which is associated with male sterility in rapid-cycling radish lines containing the Ogura cytoplasm (CrGC15) [25], and also to ascertain whether this transcript is causally related to Ogura CMS.

In order to determine the relationship between the $2700 \mathrm{nt}$ transcript and the Ogura atp $A$ locus, northern hybridizations were performed using mtRNA isolated from normal radish and fertile and sterile $\mathrm{CrGC15}$ radish plants containing the Ogura cytoplasm. A 400 bp Sal I-Bam HI fragment, internal to the atp $A$ coding region and labeled on the coding strand, hybridized to a single $1800 \mathrm{nt}$ transcript in normal radish and fertile CrGC15 mtRNA preparations, but to transcripts of $1800 \mathrm{nt}$ and $2700 \mathrm{nt}$ in sterile CrGC15 mtRNA (Fig. 3A). Experiments using a

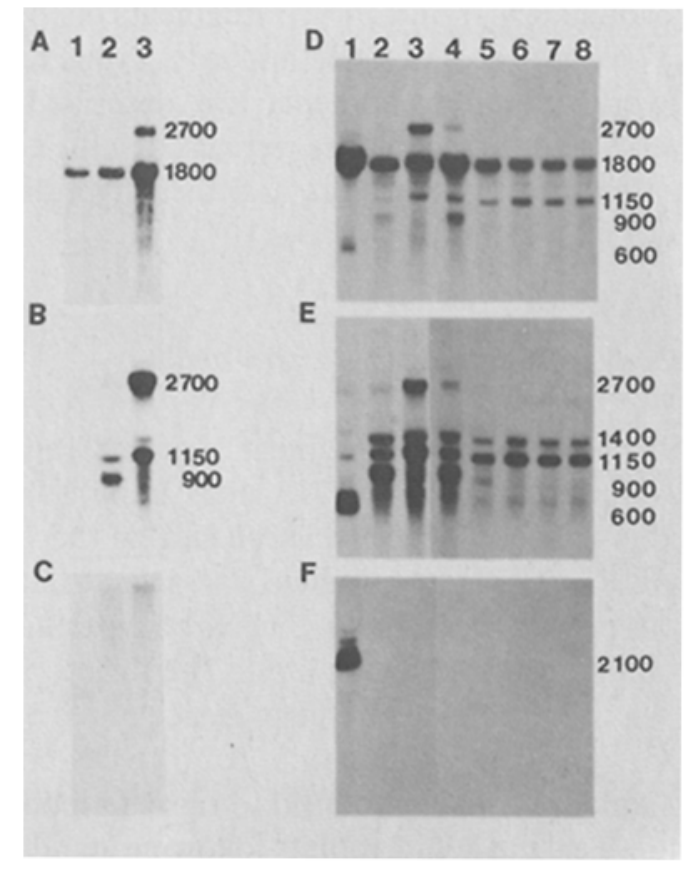

Fig. 3. Northern hybridization analysis of atpA. MtRNAs (7.5 $\mu \mathrm{g}$ each) are from normal radish (1), fertile (2) and sterile (3) CrGC15 radish, fertile (4) and sterile (5) 5241 radish, fertile (6) and sterile (7) Iwate radish, and B. napus (8) plants containing the Ogura cytoplasm. Filters were probed with the following fragments (see Fig. 1 for probe locations): a $400 \mathrm{bp}$ Sal I-Bam HI fragment internal to the atpA coding region and end-labeled at the SalI site with Klenow fragment and $\alpha-{ }^{32}$ P-dATP (panel A); a primer extension-generated fragment containing $766 \mathrm{nt}$ of coding-strand DNA from the Ogura atpA 5 ' flanking region (nucleotides -959 to -184 ) (panel B); a nick-translated plasmid containing a $2.0 \mathrm{~kb}$ Hind III-NruI fragment from the Ogura atpA $5^{\prime}$ flanking region (panel $\mathrm{C}$ ); a nick-translated plasmid containing a $1.25 \mathrm{~kb} \mathrm{Nru} \mathrm{I-SalI}$ fragment from the Ogura atpA 5 ' flanking and coding region (panel D); a $228 \mathrm{nt}$ single-stranded, $B g l$ II-Spe I (coding-strand) fragment labeled with T4 polynucleotide kinase and $\gamma^{3}{ }^{3} \mathrm{P}-\mathrm{ATP}$ and purified by strand separation (panel E), and a $228 \mathrm{nt}$ single-stranded $\mathrm{BglII}$ Spe I (noncoding-strand) fragment, which is the complement of the probe in panel $\mathrm{E}$ (panel F). Sizes are shown in nucleotides. 
probe from the Ogura atpA $5^{\prime}$ flanking region (nucleotides -950 to -184 ) identified the $2700 \mathrm{nt}$ transcript as well as Ogura-specific transcripts of $1150 \mathrm{nt}$ and $900 \mathrm{nt}$, but not the $1800 \mathrm{nt}$ transcript (Fig. 3B). A $2 \mathrm{~kb}$ probe containing sequences $5^{\prime}$ to the $\mathrm{NruI}$ site (nucleotide -950 ) failed to detect any transcripts (Fig. 3C). These results indicate that the $2700 \mathrm{nt}$ transcript originates from the Ogura atpA locus and also show that the $2700 \mathrm{nt}$ and $1800 \mathrm{nt}$ transcripts are derived from sequences $3^{\prime}$ to nucleotides -950 and -184 respectively.

We next wished to investigate the affect of nuclear background on the expression of the $2700 \mathrm{nt}$ transcript and to determine if the presence of this transcript is strictly correlated with the male-sterile phenotype. A 1282 bp Nru I-Sal I fragment containing $949 \mathrm{bp}$ and $333 \mathrm{bp}$ of Ogura atp $A 5^{\prime}$ flanking and coding regions, respectively, was used to probe northern blots of mtRNA isolated from the normal radish cytoplasm and from the Ogura radish cytoplasm in the presence of seven different nuclear backgrounds (Fig. 3D). As expected, the $1800 \mathrm{nt}$ atp $A$ transcript is present in every mtRNA preparation. The $2700 \mathrm{nt}$ transcript is detectable in mtRNA isolated from sterile CrGC15 radish plants (lane 3) and at low levels in fertile (nuclear-restored) 5241 radish plants (lane 4). This transcript is not observed in mtRNA preparations from sterile plants containing the Ogura cytoplasm and two different white radish nuclear backgrounds (lanes 5 and 7), or from sterile plants containing the Ogura cytoplasm and the B. napus nucleus (lane 8 ). This result indicates that while the presence of the $2700 \mathrm{nt}$ transcript is affected by nuclear background, its presence is not strictly correlated with the sterile phenotype.

Single-stranded probes from the Ogura atpA $5^{\prime}$ flanking region were then used to further delimit and characterize the atpA transcripts. A probe from the 5' flanking region, corresponding to the atp $A$ coding strand (nucleotides -604 to -376 ), revealed a complex transcript pattern (Fig. 3E). A $600 \mathrm{nt}$ transcript not present in any of the Ogura mtRNA preparations is detected in normal radish mtRNA. Present in all of the Ogura mtRNA preparations are transcripts of $1400 \mathrm{nt}$ and $1150 \mathrm{nt}$. A third transcript $(900 \mathrm{nt})$ is abundant in mtRNA preparations from fertile plants containing both the $\mathrm{CrGC} 15$ nucleus (lane 2) and the 5241 nucleus (lane 4). This transcript was not detected in fertile plants containing the Iwate nucleus or in any of the sterile plants. When the same region of the opposite strand (noncoding) was used as the probe in northern hybridizations, two transcripts (2100 and 2150) were identified in normal radish mtRNA (Fig. 3F). No hybridization signals are observed for any of the Ogura mtRNAs with this probe. All of the additional transcripts identified with the $228 \mathrm{bp}$, strandspecific, probes are also selected with the 1282 bp, double-stranded, Sal I-NruI probe used in Fig. 3D upon prolonged autoradiographic exposure (data not shown). In contrast to the $2700 \mathrm{nt}$ transcript, the $2150,2100,1400,1150$, 900 and $600 \mathrm{nt}$ transcripts appear to be the result of transcription originating at other regions of the genome and are therefore not directly related to atp $A$. Consistent with the theory, probes internal to the atpA coding region and $5^{\prime}$ to the $N r u$ I site (nucleotide - 994) do not detect these transcripts (Fig. 3A and 3C). In addition, the transcripts exhibit low-level hybridization signals when the Sal I-NruI fragment is used as a probe, but strong signals when small, strand-specific probes, corresponding to a repeat element present several times in both normal and Ogura mtDNAs, are used. Further experiments are necessary to determine the location of these additional transcribed regions and whether they have any coding capacity.

The $5^{\prime}$ and $3^{\prime}$ transcript termini of the 1800 and $2700 \mathrm{nt}$ transcripts were determined by $\mathrm{S} 1$ nuclease mapping experiments. The $3^{\prime}$ transcript terminus of atpA in normal radish an sterile and fertile Ogura $\mathrm{CrGC15}$ radish was mapped using a $739 \mathrm{bp}$ end-labeled Spe I-Bal I fragment (Fig. 1). One major protected fragment of 358 nucleotides was obtained from all three mtRNAs (Fig. 4D). This corresponds to a $3^{\prime}$ transcript terminus at nucleotide 1693, $172 \mathrm{nt}$ downstream from the atp $A$ coding region (Fig. 2).

The $5^{\prime}$ terminus of the $1800 \mathrm{nt}$ transcript was 
mapped using both strands of a $277 \mathrm{nt}$ Eco RI fragment ( -295 to -18 and -291 to -14$)$. The coding strand yields abundant protected fragments of 160 and $277 \mathrm{nt}$ when used as a probe with the three RNAs described above (Fig. 4A). These correspond to a major transcript terminus at -172 and fully protected probe. A major $5^{\prime}$ transcript terminus at -172 and a $3^{\prime}$ terminus at 1693 predict an atp $A$ transcript of $1865 \mathrm{nt}$; this correlates well with the $1800 \mathrm{nt}$ size estimate from northern hybridizations. While we can not eliminate the possibility that the presence of fully protected probe is the result of transcripts originating $5^{\prime}$ to the EcoRI site (-295), this seems unlikely given the presence of one predominant atp $A$ transcript (Fig. 3A) and the fact that a probe spanning the region from nucleotides -950 to -185 fails to hybridize to the $1800 \mathrm{nt}$ atp $A$

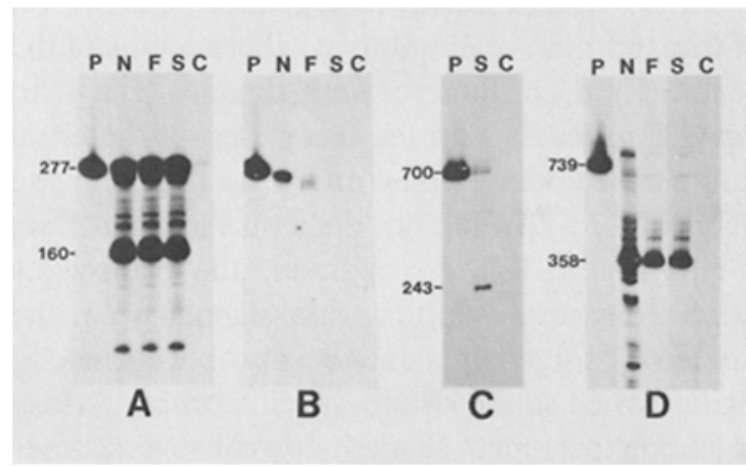

Fig. 4. Nuclease mapping of the $5^{\prime}$ and $3^{\prime}$ termini of the normal and Ogura radish atpA transcripts. Autoradiographs of nuclease mapping experiments using mtRNA $(10 \mu \mathrm{g})$ isolated from normal radish (N), and sterile (S) and fertile (F) CrGC15 plants are shown. Control lanes (C) contained $20 \mu \mathrm{g}$ of yeast tRNA. Probes (P) used were as follows: panel A, $277 \mathrm{nt}$ single-stranded EcoRI (coding strand) labeled with $\gamma_{-}{ }^{32} \mathrm{P}-\mathrm{ATP}$ and $\mathrm{T}_{4}$ polynucleotide kinase and purified by strand separation; panel $B$, the noncoding strand of the fragment in panel A; panel C, $700 \mathrm{bp} \mathrm{BglII}$ fragment from Ogura radish atpA labeled with $\gamma_{-}{ }^{32} \mathrm{P}$-ATP and $\mathrm{T}_{4}$ polynucleotide kinase; panel D, 739 bp Spe I-BalI fragments labeled at the Spe I site with $\alpha-{ }^{32} \mathrm{P}$-dATP and Klenow fragment. Nuclease mapping experiments using the $277 \mathrm{nt} E c o$ I and Spe I-Bal I fragments from both normal and Ogura radish gave identical results; this is expected because the two sequences are identical within the transcribed region. Sizes of protection fragments and probes are shown in nt next to the corresponding panel. The migration distances of fragments in different panels are not comparable. transcript (Fig. 3B). Control lanes indicate that the presence of these bands is not the result of incomplete nuclease. Rather we propose that transcripts corresponding to repeated sequences present elsewhere in the genome are responsible for complete protection of the fragment. If this is true, then other copies of the repeat must be larger than the region in common to the atp $A$ and coxI loci. Further characterization of these additional repeat elements is required to confirm this possibility. Control experiments using the noncoding strand of the EcoRI fragment as the probe resulted in no protection by the Ogura mtRNAs but low levels of fully protected probe with normal radish mtRNA (Fig. 4B). This is compatible with the finding that the noncoding strand of a repeatcontaining fragment hybridizes to transcripts in normal radish mtRNA but not Ogura radish mtRNA (Fig. 3F).

The $5^{\prime}$ end of the $2700 \mathrm{nt}$ Ogura atp $A$ transcript was mapped using a $700 \mathrm{bp}$ end-labeled $B g l$ II fragment, extending from a $B g l$ II site approximately $350 \mathrm{bp} 5^{\prime}$ to the characterized region to nucleotide -600 . When this fragment was used as a probe with sterile Ogura radish RNA, a protected fragment of $243 \mathrm{nt}$ as well as low levels of fully protected probe were obtained (Fig. 4C). The $243 \mathrm{nt}$ protected fragment corresponds to a 5' transcript terminus at -843 (Fig. 2) and predicts an Ogura atpA transcript of $2536 \mathrm{nt}$. This is slightly smaller than the predicted size of $2700 \mathrm{nt}$ (hereafter referred to as the $2536 \mathrm{nt}$ transcript) based on northern hybridization results. The presence of fully protected probe could be due to either transcription originating $5^{\prime}$ to the first $B g l$ II site or to the fact that a doublestranded probe was used in the nuclease mapping experiments. That a northern hybridization using a probe extending $5^{\prime}$ to the $N r u$ I site (nucleotide -994) failed to detect the $2536 \mathrm{nt}$ transcript (Fig. 3C) suggests that -843 is in fact the $5^{\prime}$ terminus of this transcript.

Several conclusions can be drawn from the transcript mapping experiments. 1) The $2536 \mathrm{nt}$ Ogura atp $A$ transcript results from transcription originating upstream of the $5^{\prime}$ rearrangement breakpoint and extending into the atp $A$ coding 
region. 2) A repeated sequence present in the $5^{\prime}$ flanking region of atp $A$ and implicated in mtDNA rearrangements is transcribed elsewhere in the normal and Ogura radish mitochondrial genomes. 3) Although the $2536 \mathrm{nt}$ Ogura atp $A$-associated and $900 \mathrm{nt}$ repeat-associated transcripts are affected by nuclear background, neither maintain a strict correlation with the sterility/fertility phenotype when a large number of nuclear backgrounds are examined. This indicates that they are probably not causally related to Ogura CMS.

Ogura radish mitochondria contain normal levels of ATPA polypeptides

In order to determine if either the $2536 \mathrm{nt}$ transcript or nuclear background affect the level of the ATPase $\alpha$-subunit, antibodies were raised to the $\alpha$-subunit and the size and relative amount of the polypeptide determined for several nuclear/mitochondrial combinations. An example of the

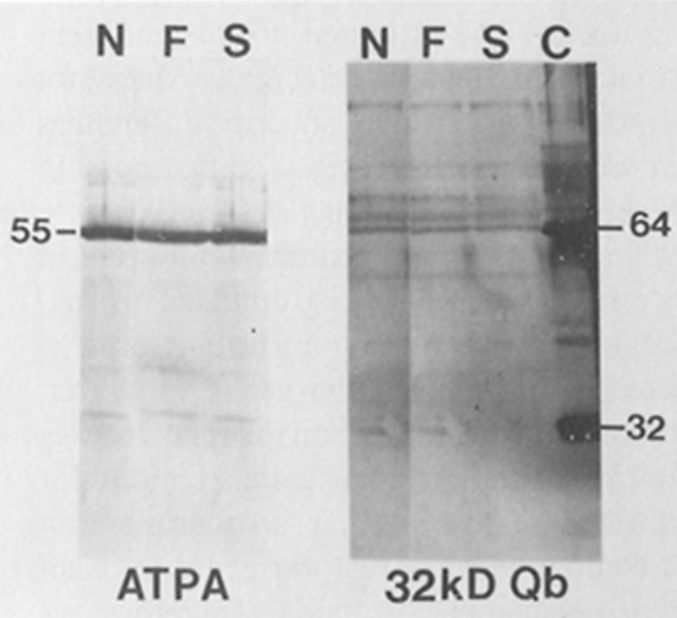

Fig. 5. Immunodetection of ATPA in normal and Ogura mitochondria. Western blots containing mitochondrial proteins $(200 \mu \mathrm{g})$ from normal radish (N) and sterile (S) and fertile (F) CrGC15 plants as well as chloroplast proteins (C) from normal radish were treated with antisera raised against an ATPA- $\beta$-galactosidase fusion protein or the spinach chloroplast $p s b A$-encoding $32 \mathrm{kDa}$ protein. The $32 \mathrm{kDa}$ protein dimerizes to give a $64 \mathrm{kDa}$ protein band. Sizes are shown in $\mathrm{kDa}$. results from these experiments is shown in Fig. 5. A polypeptide of approximately $55000 \mathrm{Da}$ is detected in western blots of normal radish $(\mathrm{N})$ and fertile $(\mathrm{F})$ and sterile $(\mathrm{S}) \mathrm{CgGC15}$ radish mitochondrial proteins with the anti- $\alpha$-subunit antibody. The same relative amount of $\alpha$-subunit is detected in all three mitochondrial preparations. Similar levels of ATPase $\alpha$-subunit were found in mitochondria isolated from all eight different nuclear backgrounds (data not shown). The lack of cross-reaction between the three mitochondrial preparations and antibody to the spinach chloroplast $32 \mathrm{kDa} \mathrm{Qb}$ (quinone-binding) protein indicates that there is little or no chloroplast contamination in the mitochondrial preparations and that all of the ATPase $\alpha$-subunit cross-reactivity is due to mitochondrial protein. Likewise, equal levels of cross-reactivity are observed in all three mitochondrial preparations when western blots are treated with antibody to subunit II of the maize cytochrome oxidase (data not shown). By demonstrating that neither nuclear background nor cytoplasm affects the size or level of the $\alpha$-subunit, these results support those obtained from DNA sequence analysis and transcript mapping experiments, which indicate that atp $A$ is not associated with Ogura CMS.

\section{Discussion}

We have characterized the atpA locus from both the normal and Ogura cytoplasms of radish to investigate rearrangements associated with atp $A$ and also to determine if a $2700 \mathrm{nt}$ (actual size $2536 \mathrm{nt}$ ) atp $A$ transcript that we had previously found associated with sterile plants containing the Ogura cytoplasm could be causally related to Ogura CMS. Several lines of evidence indicate that the atpA locus and the $2536 \mathrm{nt}$ transcript are not involved in male sterility. 1) The two loci are virtually identical $(99.8 \%$ ) over a $3.8 \mathrm{~kb}$ region that encompasses a 507 codon opening reading frame, whose product is approximately $94 \%$ identical with other plant mitochondrial ATPA polypeptides. 2) The predominant atp $A$ transcript $(1865 \mathrm{nt})$ is completely contained within the 
unrearranged region of the locus. In addition, there are no DNA-encoded open reading frames or other structures in the Ogura atp $A 5^{\prime}$ flanking region that can be implicated in male sterility. However, we can not eliminate the possibility that the RNA is edited and that the transcript contains an open reading frame not found in the DNA. 3) Analysis of atpA transcript patterns in several different sterile/fertile lines indicated that while the $2536 \mathrm{nt}$ transcript is affected by nuclear background, it is not strictly correlated with male sterility. 4) Sterile and fertile plants possessing the Ogura cytoplasm contain an ATPA polypeptide of the same size and abundance as do normal radish plants.

The 5 ' rearrangement breakpoint between the normal and Ogura atpA loci occurs 613 bp $5^{\prime}$ to the coding region and within a $689 \mathrm{bp}$ repeated sequence (nucleotides -855 to -166 , normal radish) that is also present in its entirety $5^{\prime}$ to normal radish coxI-2, a submolar form of the gene. This repeated sequence has been implicated in the homologous rearrangement events that generate the coxI-2 locus (Makaroff, Apel and Palmer, unpublished). Included in this larger repeat, and immediately flanking the $5^{\prime}$ points of divergence between the normal and Ogura atpA loci as well as the normal radish coxI- 1 and coxI-2 loci, is a 156 bp sequence that is also present $5^{\prime}$ to the orf105-atp6 locus in Ogura radish [25]. Much of this region (122 bp) is homologous to a 175 bp sequence common to the $5^{\prime}$ flanking regions of tobacco atp 6 and $r p s 13[4,5]$ and Oenothera atp6, coxI and coxIII $[15,36]$. The Oenothera coxI- and coxIII-linked copies of the repeat are part of a $657 \mathrm{bp}$ sequence that has been implicated in both promoter function and mtDNA rearrangements [15]. Further work is necessary to determine what role, if any, this sequence, which is found $5^{\prime}$ to genes in several diverse plants and within larger repeats that have been implicated in recombination events, actually plays in plant mtRNA structure and/or function.

Transcript mapping experiments indicate that the $2536 \mathrm{nt}$ atpA transcript found associated with sterile CrGC15 plants is the result of transcription originating $5^{\prime}$ to the abundant $1865 \mathrm{nt}$
atpA transcript. The $2536 \mathrm{nt}$ and $1836 \mathrm{nt}$ transcripts could be related in two ways: 1 ) they could represent two distinct transcriptional units and nuclear background controls either transcription initiation or transcript stability or 2) the $1836 \mathrm{nt}$ transcript could be a processed form of the $2536 \mathrm{nt}$ transcript and nuclear background controls the level or activity of a RNA processing enzyme. Further experiments are required before we can distinguish between these possibilities. The $2536 \mathrm{nt}$ transcript is not present in sterile plants containing three other nuclear backgrounds suggesting that the gene(s) that affect this transcript are not restorer genes.

The $900 \mathrm{nt}$ transcript, which contains a repeat, is also affected by nuclear background (Fig. 3E). This transcript is present in fertile plants containing the Ogura cytoplasm in combination with either the CrGC15 nucleus or the 5241 nucleus. It is not present in normal radish plants, fertile Iwate plants, or in sterile Iwate, $5241, \mathrm{CrGC} 15$ or $B$. napus plants containing the Ogura cytoplasm. Like the $2536 \mathrm{nt}$ transcript, it is not clear at what level the nuclear-encoded factor acts. However, no differences in restriction fragment profiles of Ogura mitochondria isolated from different nuclear backgrounds are detectable (data not shown), indicating that the transcript differences are most likely not the result of differences in mtDNA genome organization.

The $900 \mathrm{nt}$ transcript is found in only two of the three restored nuclear backgrounds. This suggests that, similar to the $2536 \mathrm{nt}$ transcript, it is not affected by nuclear restorer genes. However, beause there is no information on the relative roles of the multiple nuclear restorer genes reported for the Ogura cytoplasm [14], it is possible that different combinations of restorer genes may act in different nuclear backgrounds. Therefore, we can not rule out the possibility that the 2536 or $900 \mathrm{nt}$ transcripts may play some role in Ogura CMS that is not related to atpA itself.

Nuclear genes distinct from those that control fertility restoration have also been shown to affect the levels of the urf13-T and orf 25 transcripts in the CMS-T cytoplasm of maize [17]. In addition, nuclear effects on the translation of several mito- 
chondrial protein in sorghum [1] and TURF-13 in the CMS-T cytoplasm of maize [9] have been observed. The primary effect of the maize nuclear RF1 gene appears to be post-transcriptional, acting as an RNA processing enzyme or a transcriptional or translational factor $[10,16]$. While a number of nuclear genes that are involved in yeast mitochondrial biogenesis (including DNA replication, transcription and translation factors) have been isolated and characterized [reviewed in 40], we are just beginning to characterize processes involved in plant mitochondrial biogenesis.

We have reported here the identification of two mitochondrial transcript differences in the Ogura cytoplasm of radish that appear to reflect the action of at least two nuclear loci. Further studies are necessary to determine whether these loci act at the level(s) of transcription initiation, RNA processing or RNA stability and whether they play any role in the regulation of mitochondrial function.

\section{Acknowledgements}

This work was supported by grants R01 GM 35087 (to J. D. P.) and F32 GM 11323 (to C. A. M.) from the National Institute of Health. We thank M. Temple for critical reading of the manuscript, D. Bay for photographic services, N. Bowlby and C. S. Levings III for antisera and S. Magnuson and J. Imamura for the Ogura CMS lines.

\section{References}

1. Bailey-Serres J, Dixon LK, Liddell AD, Leaver CJ: Nuclear-mitochondrial interactions in cytoplasmic malesterile Sorghum. Theor Appl Genet 73: 252-260 (1986).

2. Berk AJ, Sharp PA: Sizing and mapping of early adenovirus $\mathrm{mRNAs}$ by gel electrophoresis of $\mathrm{S} 1$ endonuclease digested hybrids. Cell 12: 721-732 (1977).

3. Birnboim $\mathbf{H C}$, Doly $\mathbf{J}$ : A rapid alkaline extraction procedure for screening recombinant plasmid DNA. Proc Natl Acad Sci USA 76: 1513-1523 (1979).

4. Bland MM, Levings CS III, Matzinger DF: The tobacco mitochondrial ATPase subunit 9 gene is closely linked to an open reading frame for a ribosomal protein. Mol Gen Genet 204: 8-16 (1986).
5. Bland MM, Levings CS III, Matzinger DF: The ATPase subunit 6 gene of tobacco mitochondria contains an unusual sequence. Curr Genet 12: 475-481 (1987).

6. Braun CJ, Levings CS III: Nucleotide sequence of the F1-ATPase $\alpha$ subunit gene from maize mitochondria. Plant Physiol 79: 571-577 (1985).

7. Chaumont F, Boutry $\mathbf{M}$, Briquet $M$, Vassarotti A: Sequence of the gene encoding the mitochondrial F1-ATPase alpha subunit from Nicotiana plumbaginifolia. Nucleic Acids Res 16: 6247 (1988).

8. Covello PS, Gray MW: RNA editing in plant mitochondria. Nature 341: 662-666 (1989).

9. Dewey RE, Levings CS III, Timothy DH : Novel recombinations in the maize mitochondrial genome produce a unique transcriptional unit in the Texas male-sterile cytoplasm. Cell 44: 439-449 (1986).

10. Dewey RE, Schuster AM, Levings CS III, Timothy DH: Nucleotide sequence of $\mathrm{F}_{0}$-ATPase proteolipid (subunit 9) gene of maize. Proc Natl Acad Sci USA 82: 1015-1019 (1985).

11. Gualberto JM, Lamattina L, Bonnard G, Weil J-H, Grienberger J-M: RNA editing in wheat mitochondria results in convension of protein sequences. Nature 341 : 660-662 (1989).

12. Harlow E, Lane D: Antibodies: A laboratory manual, Cold Spring Harbor Laboratory, Cold Spring Harbor, NY (1988).

13. Henikoff $S$ : Unidirectional digestion with exonuclease III creates targeted breakpoints for DNA sequencing. Gene 28: 351-359 (1984).

14. Heyn FW: Introgression of restorer genes for Raphanus sativus into cytoplasmic male sterile Brassica napus and the genetics of fertility restoration. Proc 5th Int Rapeseed Conf (Malmö). 82-83 (1978).

15. Hiesel R, Schobel W, Schuster W, Brennicke A: The cytochrome oxidase subunit I and subunit III genes in Oenothera mitochondria are transcribed from identical promoter sequences. EMBO J 6: 29-34 (1987).

16. Kennel JC, Pring RD: Initiation and processing of atp6, urf13 and ORF221 transcripts from mitochondria of $\mathrm{T}$ cytoplasm of maize. Mol Gen Genet 216: 16-24 (1989).

17. Kennel JC, Wise RP, Pring RD: Influence of nuclear background on transcription of a maize mitochondrial region associated with Texas male sterile cytoplasm. Mol Gen Genet 210: 399-406 (1987).

18. Kolodner R, Tewari KK: Physicochemical characterication of mitochondrial DNA from pea leaves. Proc Natl Acid Sci USA 69: 1830-1834 (1972).

19. Laemmii EK: Cleavage of structural proteins during the assembly of the head of bacteriophage T4. Nature 227 : 680-685 (1970).

20. Leaver CJ, Hack E, Forde BG: Protein synthesis by isolated plant mitochondria. Methods Enzymol 97: 476-484 (1983).

21. Levings CS III, Dewey RE: Molecular studies of cytoplasmic male sterility in maize. Phil Trans $\mathrm{R}$ Soc Lond B 319: 177-185 (1988). 
22. Lonsdale DM: Cytoplasmic male sterility: a molecular perspective. Plant Physiol Biochem 25: 265-271 (1987).

23. Lowry OH, Rosebrough NT, Farr AL, Randall RJ: Protein measurement with the Folin phenol reagent. J Biol Chem 193: 265-275 (1951).

24. Makaroff CA, Apel IJ, Palmer JD: The atp6 coding region has been disrupted and a novel reading frame generated in the mitochondrial genome of cytoplasmic male-sterile radish. J Biol Chem 264: 11706-11713 (1989).

25. Makaroff CA, Palmer JD: Mitochondrial DNA rearrangements and transcriptional alterations in the male-sterile cytoplasm of Ogura radish. Mol Cell Biol 8: 1474-1480 (1988).

26. Makaroff CA, Palmer JD: Extensive mitochondrial specific transcription of the Brassica campestris mitochondrial genome. Nucleic Acids Res 15: 5141-5156 (1987).

27. Maniatis T, Fritsch EF, Sambrook J: Molecular cloning: A laboratory manual. Cold Spring Harbor Laboratory, Cold Spring Harbor, NY (1982).

28. Morikami A, Nakamura K: Structure and expression of pea mitochondrial F1 ATPase $\alpha$-subunit gene and its pseudogene involved in homologous recombination. J Biochem 101: 967-976 (1987).

29. Ogura H: Studies on the new male-sterility in Japanese radish, with special references to the utilization of this sterility towards the practical raising of hybrid seeds. Mem Fac Agric Kagoshima Univ 6: 39-78 (1968).

30. Palmer JD: Intraspecific variation and multicircularity in Brassica mitochondrial DNAs. Genetics 118: 341-351 (1988).
31. Palmer JD, Herbon LA: Tripartite mitochondrial genomes of Brassica and Raphanus: reversal of repeat configurations by inversion. Nucleic Acids Res 14: 9755-9765 (1986).

32. Pelletier G, Vedel F, Belleard G: Cybrids in genetics and breeding. Hereditas (suppl) 3: 49-56 (1985).

33. Ruther V, Muller-Hill B: Easy identification of cDNA clones. EMBO J 2: 1791-1794 (1983).

34. Sanger F, Nicklen S, Coulson AR: DNA sequencing with chain-terminating inhibitors. Proc Natl Acad Sci USA 74: 5463 (1977).

35. Schuster W, Brennicke A: Pseudocopies of the ATPase $\alpha$-subunit gene in Oenothera mitochondria are present on different circular molecules. Mol Gen Genet 204: 29-35 (1986).

36. Schuster W, Brennicke A: Nucleotide sequence of the Oenothera ATPase subunit 6 gene. Nucleic Acids Res 15: 9092 (1987).

37. Schuster W, Wissinger B, Unseld $\mathbf{M}$, Brennicke A: Transcripts of the NADH-dehydrogenase subunit 3 gene are differentially edited in Oenothera mitochondria. EMBO J 9: 263-269.

38. Stern DB, Newton KJ: Isolation of plant mitochondrial RNA. Methods Enzymol 118: 488-496 (1986).

39. Towbin H, Staehelin T, Gorden J: Electrophoretic transfer of proteins from polyacrylamide gels to nitrocellulose sheets; Procedure and some applications. Proc Natl Acad Sci USA 76: 4350-4354 (1979).

40. Tzagoloff A, Meyers AM: Genetics of mitochondrial biogenesis. Ann Rev 